\title{
A Neural Network Approach to Real-Time Condition Monitoring of Induction Motors
}

\author{
Mo-yuen Chow, Member, IEEE, Peter M. Mangum, Member, IEEE, and \\ Sui Oi Yee, Student Member, IEEE
}

\begin{abstract}
Induction motors are subject to incipient faults which, if undetected, can lead to serious machine failures. Incipient fault detection methods applied in large induction motors of greater than $100 \mathrm{hp}$ are often too costly or physically impractical for use on small- and medium-size induction motors. This paper develops a neural network-based incipient fault detector for small- and medium-size induction motors. The neural network-based incipient fault detector avoids the problems associated with traditional incipient fault detection schemes by employing more readily available information such as rotor speed and stator current. The neural network design is evaluated in real time in the laboratory on a $\frac{3}{4}$-hp permanent magnet induction motor. The results of this evaluation indicate that the neural network-based incipient fault detector provides a satisfactory level of accuracy, greater than $95 \%$, which is suitable for real-world applications.
\end{abstract}

\section{INTRODUCTION}

$\mathrm{T}$ HE simple, robust design and construction of ac induction motors have encouraged their successful application in industry for many years. As with all types of rotating machines, however, ac induction motors are subject to the occurrence of incipient faults [1]-[6]. If left undetected, the occurrence of an incipient fault will eventually degenerate into a machine failure. Despite the potential for serious failures in all sizes of electric rotating machines, traditional methods of incipient fault detection applied in large electric machines are generally not applied in smaller machines due to limitations related to sensing-device size and economic considerations.

The objective of this research has been to develop an alternative to traditional incipient fault-detection schemes that overcome the limitations of the present technology. The squirrel-cage type ac induction motor is chosen as a prototype-rotating machine for the incipient fault study and incipient fault-detector application. The basic requirement of the neural network-based incipient fault detection scheme is the ability to detect the occurrence of an incipient fault within an induction motor in real time.

Previous investigations on the application of neural net-

Manuscript received November 1, 1990; revised May 28, 1991 and August 14, 1991. This paper was supported by the National Science Foundation under Grant No. ECS-8922727 and the Electrical Power Research Center Project Grant 5-30970 of North Carolina State University.

M. Chow and S. O. Yee are with the Department of Electrical Engineering, North Carolina State University, Raleigh, NC 27695.

P. M. Mangum is with Control Products Business, Square D Co., Raleigh, NC 27611 .

IEEE Log Number 9103486. works to the problem of detecting the occurrence of incipient faults in rotating electric machines have been made [3], [4]. In [3], computer simulations of induction motors and of neural networks demonstrate the feasibility of a neural network-based incipient fault detector as applied to the problem of stator winding and rotor-bearing incipient faults. This paper presents the work and the results of using the neural network detector for an actual induction motor in real time to show the actual feasibility of using artificial neural networks in a real industrial situation. Different network structures, performances, and training time required are presented and discussed in the paper. Results are presented that indicate the success of the incipient fault detector in laboratory testing and indicates its promising future for industrial usages. The final section concludes with a summary of the present achievements.

\section{BRIEF Description of the InCIPIENT Fault Problem and Neural Network Detection TECHNOLOGY}

This paper investigates two of the most common types of incipient faults in a single-phase squirrel-cage induction motor: stator winding fault and bearing wear under constant load torque conditions. The stator winding, the most vulnerable component of the squirrel-cage type ac induction motor, is subject to shorted turns caused by deterioration and failure of the stator winding insulation due to excessive temperature, age, or vibration [1], [2], [5]. Traditional methods for monitoring the condition of stator windings include regularly scheduled motor inspections and the use of temperature-sensing devices embedded within the motor windings. Unfortunately, regularly scheduled motor inspections often prove too costly to be performed for small- or medium-size motors of less than $100 \mathrm{hp}$. Temperature-sensing devices and associated components are also relatively expensive and may not be physically applicable in the smaller size motors.

The rotor bearings of the induction motor are subject to deterioration caused by inadequate or contaminated lubrication, misapplication, or misalignment. Traditionally, rotorbearing temperature and vibration levels have been monitored in order to estimate rotor-bearing condition [1], [2]. As with the stator winding, temperature-sensing devices for the rotor bearings are limited to application in large motors of $100 \mathrm{hp}$ or greater due to cost and physical size constraints. Similarly, the cost of vibration-sensing devices and associated signalprocessing hardware limits the use of these devices in large machines only. 
The methodology and performance of the use of artificial neural networks to detect incipient faults in a single-phase squirrel-cage induction motor have been presented in [3] and [4] and are briefly described in this section for completeness. Two specific faults of single-phase induction motors, i.e., turn-to-turn insulation faults and bearing wear, are investigated. The motor is assumed to operate under a known load torque at a steady-state condition. A turn-to-turn insulation incipient fault in the main winding of the motor will cause a change in the corresponding number of equivalent turns $N$ of the main winding. Likewise, bearing wear of the motor is reflected in the damping coefficient $B$ of the motor. To determine the most suitable motor measurements for detecting the incipient faults under consideration, in terms of easy accessibility, reliability and sensitivity, the dynamics of induction motors were analyzed. From the analysis, the steady-state current $I$ and the rotor speed $\omega$ of the motor can be related to the main winding equivalent turns $N$ and the damping coefficient $B$ by a nonlinear equation:

$$
g(I, \omega, N, B)=\mathbf{0}
$$

where $g=\left[g_{1}, g_{2}\right]^{T}$. In this paper, values of $I$ and $\omega$ are bounded such that $\{2 \mathrm{~A} \leq I \leq 15 \mathrm{~A}\}$ and $\{100 \mathrm{rad} / \mathrm{s} \leq \omega \leq$ $188 \mathrm{rad} / \mathrm{s}\}$, respectively, for acceptable operating conditions of the motor under investigation. For a more detailed induction motor dynamics derivation and analysis, see [3].

Equation (1) suggests that indications of the condition of the winding and bearing can be obtained from measurements of the stator current and rotor speed. Indeed, from analysis, the stator current $I$ and the rotor speed $\omega$ are found to be very sensitive to the changing conditions of the stator winding and bearings. Moreover, the current and speed are easily accessible and can be measured quite accurately. Therefore, for the detection of turn-to-turn insulation faults and bearing wear in single-phase induction motors, the inputs to the neural network-based fault detector are $\{I, \omega\}$-the stator current and rotor speed. The desired network outputs are $\left\{N_{c}, B_{c}\right\}$, the qualitative conditions of the winding insulation and bearing wear.

Define $\mathscr{M}_{1}$ as the mapping from $\{I, \omega\}$ to $\{N, B\}$ :

$$
\mathscr{M}_{1}:\{I, \omega\} \rightarrow\{N, B\} \text {. }
$$

As previously stated, the conditions of the main winding and the bearing of the motor are reflected in the numerical values of the main winding equivalent turns $N$ and the damping coefficient $B$, respectively. For the specific induction motor application under study, the conditions of $N_{c}$ and $B_{c}$ are then quantized into three condition levels (good, fair, bad) based on the values of $N$ and $B . \mathscr{M}_{2}$ is used to denote the mapping of the quantization of $\{N, B\}$ to $\left\{N_{c}, B_{c}\right\}$ :

$$
\mathscr{M}_{2}:\{N, B\} \rightarrow\left\{N_{c}, B_{c}\right\} .
$$

As a result, the mapping $\mathscr{M}$ from $\{I, \omega\}$ to $\left\{N_{c}, B_{c}\right\}$ can be written as a composition of $\mathscr{M}_{1}$ and $\mathscr{M}_{2}$ :

$$
\mathscr{M}=\mathscr{M}_{1} \cdot \mathscr{M}_{2}:\{I, \omega\} \rightarrow\left\{N_{c}, B_{c}\right\} .
$$

Mapping $\mathscr{M}$ is very complex due to the high degree of nonlinearity of the induction motor dynamics and of the discretization involved; thus obtaining an accurate analytical expression for $\mathscr{M}$ for a given induction motor is rather difficult. However, this complexity can be avoided by training a neural network to learn the desired mapping of $\mathscr{M}$ based solely on input-output examples that can be obtained accurately. An artificial neural network is composed of highly interconnected units (neurons) with a deterministic, semilinear activation function. The neural network is trained by adjusting the numerical values of the weights-or network interconnections-between each unit [7]-[9]. Once the neural network is appropriately trained, the network weights will contain the nonlinearity of the desired mapping, so that the difficulties of mathematical modeling can be avoided.

The development of the neural network-based incipient fault detector for the induction motor requires training data for classifying the relative condition of the stator winding with respect to the presence, or absence, of an incipient fault. The classification is usually a fuzzy logic task and is supplied by an expert according to his or her experience. For this research, two cost functions are developed as criteria to evaluate the conditions of winding and bearing, respectively, in order to quantitatively validate the performance of the artificial neural network fault detection. In actual applications of the incipient fault detector, the cost functions are not required and the criteria are chosen based on the operating environment supplied by experts.

A decrease in the winding equivalent turns will increase stator winding current, thus causing increased heating in the stator due to additional $I^{2} R$ losses. The increased heating will cause a corresponding temperature rise in the stator, thereby decreasing the life expectancy of the stator winding insulation. Stator winding insulation failure will cause additional shorted turns, further increases in temperature, and a further increase in the rate of deterioration of the stator winding insulation. If left unchecked, this process will cause the eventual destruction of the stator winding and render the motor inoperable. A criterion based on the relationship between stator currents, motor temperature, and insulation life suggested in [5], [6] is used to evaluate the stator winding condition.

The frictional losses within the motor bearings is used as the criterion to determine their condition. The bearings can provide losses that are negligible or within $5 \%$ of the overall losses of the motor and be considered good. Bad bearings, however, can cause permanent damage to themselves as well as to the rotor or the stator windings and must be detected quickly. Using efficiency and motor nameplate data, an estimate of bearing losses, and relative bearing condition are obtained and used to determine the bearing condition.

The inputs to the incipient fault detector consists of two signals corresponding to steady-state stator winding rms current $I$ and rotor shaft speed $\omega$. The outputs of the incipient fault detector are the condition of the stator winding $N_{c}$ and the condition of the motor bearings $B_{c}$. Although the activation of the output nodes is a continuous function, the training data sets present discrete target values of $0.9,0.5$, and 0.1 to the neural network, which correspond to relative conditions of good, fair, and poor, respectively. Classifying functions 
that are external to the neural network are provided for the nearest neighborhood discretization of the continuous valued output activation function when the incipient fault detector is used for actual fault detection purposes.

\section{Laboratory Testing of the Neural Network INCIPIENT FAULT DETECTOR}

The performance of the neural network based incipient fault detector was tested using the laboratory test stand shown schematically in Fig. 1. This laboratory test stand consists of a Dayton model 6K624B $\frac{3}{4}$-hp split-phase ac induction motor mechanically coupled to a $\frac{3}{4}$-hp permanent magnet dc motor. A pulse tachometer and a Hall effect current-sensing device provide operating data to an IBM PC compatible computer via a general purpose analog to digital $\mathrm{I} / \mathrm{O}$ board installed in the computer. IFDNN represents an incipient fault detection via neural network (RT_IFDNN is the real-time version of IFDNN) and will be discussed in more detail in later sections.

The ac induction motor is a split-phase dual-voltage motor that is configured to operate at $115 \mathrm{~V}$ ac. The stator winding has been modified by addition of external wires attached to 10 of the stator winding end turns, as shown in Fig. 2. The other end of these external wires is connected to a terminal strip located near the motor. By short circuiting one or more pairs of these external connections at the terminal strip, it is possible to emulate stator winding turn-to-turn faults. The construction of the stator of the induction motor is such that it is impossible to determine the interrelationships of the modified windings by visual inspection. Therefore, the method of determining winding relationships consisted of measuring the voltage drop between the external connection points while the motor was operating under no-fault conditions.

The incipient fault detection experiments were conducted using only single-turn short circuits of the stator winding. Faults consisting of more than a single turn are created by constructing several single-turn short circuits. Multiple-turn short circuits were not used due to the high level of current measured in the shorting jumpers. High-current levels can easily damage the stator winding, especially in the locations where the modifications are present.

A dc motor coupled to the induction motor is used. There are two purposes of this dc motor. First, by operating the dc motor as a generator supplying a constant current load, the motor provides a constant torque load to the shaft of the ac induction motor. Thus a constant armature current provides the constant load for simulation of normal operating conditions. Second, by increasing the constant current load above the normal operating point, the dc motor also provides a variable torque load corresponding to the additional loading caused by a worn bearing in the ac induction motor. This allows nondestructive emulation of worn or failed induction motor bearings.

The induction motor rotor speed is sensed by a pulse tachometer mounted, for convenience, to the face of the dc motor. The rotor speed input to the IBM PC compatible computer is provided via a frequency input to the general purpose I/O board located within the computer. The induc-

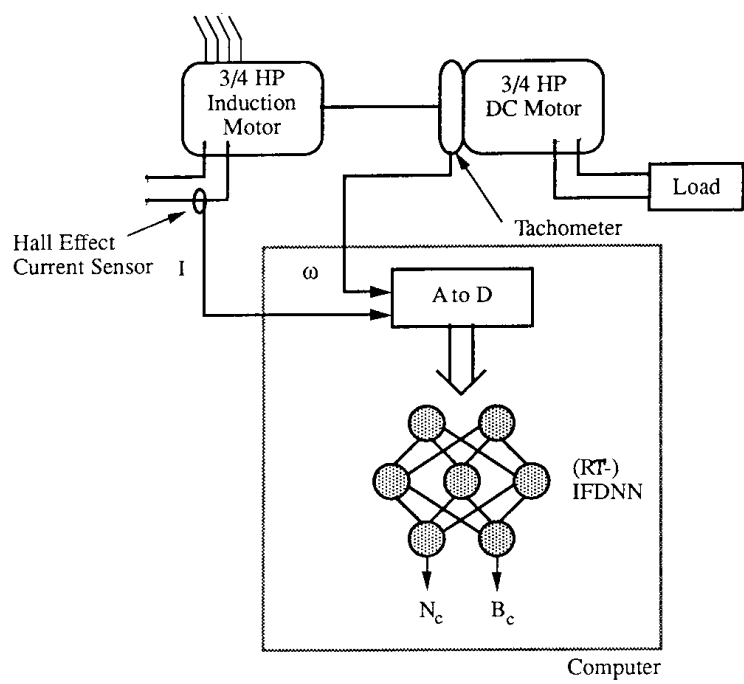

Fig. 1. Setup of the incipient fault detection test stand in the laboratory.

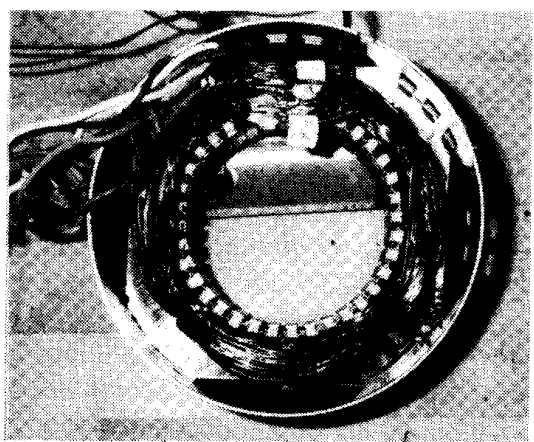

Fig. 2. Modification for insulation failure simulation in the stator winding

tion motor stator winding current is sensed using a Hall-effect device. One wire of the main power source to the induction motor is looped through the Hall effect device. The output of this device is measured via an analog input to the general purpose I/O board located within the IBM PC compatible computer. The output of the Hall effect device is the instantaneous value of the stator current. Consequently, a conversion to rms stator current is provided in the software.

The neural network-based incipient fault detectors are implemented in the $\mathrm{C}$ programming language on an IBM PC compatible computer. The softwares are termed IFDNN and RT_IFDNN. IFDNN is an implementation of the generic three-layer feed-forward neural network of Fig. 3 as well as the higher order neural network of Fig. 4. The structure of the neuron used in IFDNN is shown in Fig. 5. The variable $j$ represents the $j$ th neuron, $n$ is the number of inputs $x=$ $\left[x_{1}, \ldots, x_{n}\right]^{T}$ to the $j$ th neuron, and $\boldsymbol{w}=\left[w_{j 1}, \ldots, w_{j n}\right]$ and $\theta_{j}$ are the weights and internal threshold, respectively, of the $j$ th neuron to be adjusted throughout the training session. The neuron summation is

$$
s_{j}=\sum_{i=1}^{n} w_{j i} x_{i}+\theta_{j}
$$

The sigmoid function is used as the activation function $f$ so 


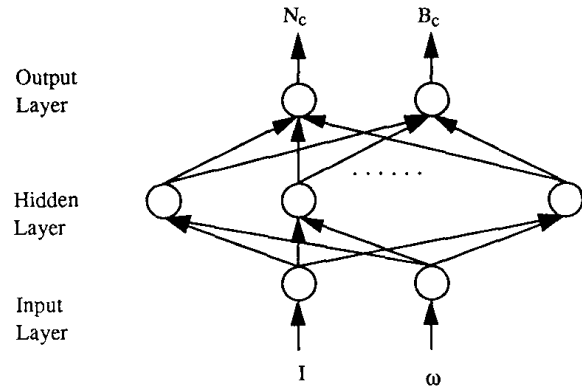

Fig. 3. Structure of the conventional IFDNN

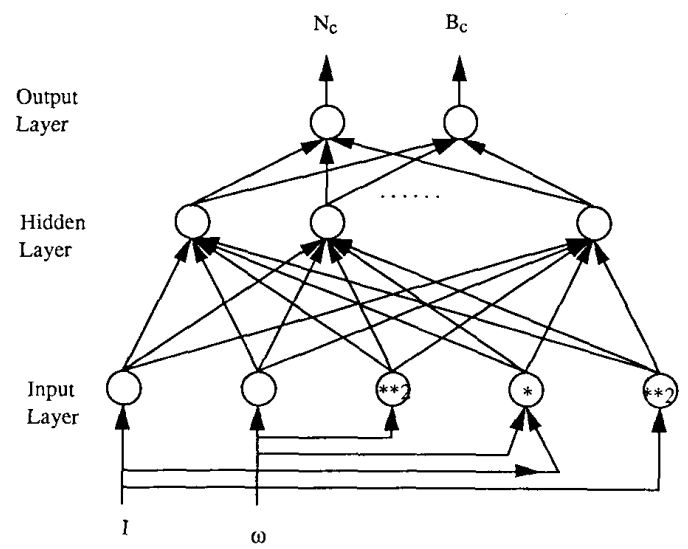

Fig. 4. Structure of the higher order IFDNN.

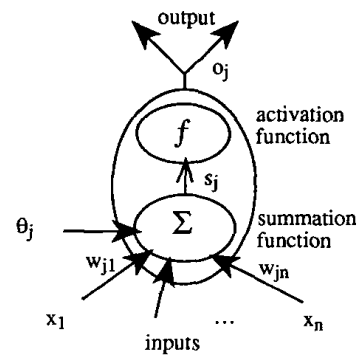

Fig. 5. Structure of the neuron structure used in IFDNN.

the output of a neuron can be written as

$$
o_{j}=f\left(s_{j}\right)=\frac{1}{1+e^{-s_{j}}} .
$$

The back-propagation training algorithm is used to adjust the numerical values of the weights and the internal threshold of each neuron [7]-[9]. The network is trained by initially selecting small random weights and internal thresholds and then presenting all training data repeatedly. Weights and thresholds are adjusted after every training example is presented to the network, until weights converges or the twonorm error is reduced to an acceptable value.

Note that in the higher order network structure (Fig. 4) the original input space $\{I, \omega\}$ (Fig. 3) is expanded to five dimensions $\left\{I, \omega, I^{2}, \omega^{2}, I^{*} \omega\right\}$ to achieve better network accuracy and to reduce training time [3]. The software
TABLE I

Set of Training Examples Used to Train IFDNN

\begin{tabular}{|c|c|c|c|}
\hline$I(\mathrm{pu})$ & $\omega(\mathrm{pu})$ & $N_{c}$ & $B_{c}$ \\
\hline 0.00000 & 0.74359 & 0.900 & 0.900 \\
\hline 0.07000 & 0.53846 & 0.900 & 0.900 \\
\hline 0.14000 & 0.38462 & 0.900 & 0.500 \\
\hline 0.21000 & 0.25641 & 0.900 & 0.100 \\
\hline 0.28000 & 0.05128 & 0.900 & 0.100 \\
\hline 0.02000 & 0.87180 & 0.900 & 0.900 \\
\hline 0.08000 & 0.64103 & 0.900 & 0.900 \\
\hline 0.15000 & 0.53846 & 0.900 & 0.500 \\
\hline 0.22000 & 0.35897 & 0.900 & 0.100 \\
\hline 0.29000 & 0.17949 & 0.900 & 0.100 \\
\hline 0.03000 & 0.89744 & 0.900 & 0.900 \\
\hline 0.11000 & 0.71795 & 0.900 & 0.900 \\
\hline 0.17000 & 0.58974 & 0.900 & 0.500 \\
\hline 0.24000 & 0.41026 & 0.900 & 0.100 \\
\hline 0.31000 & 0.23077 & 0.900 & 0.100 \\
\hline 0.10000 & 1.00000 & 0.500 & 0.900 \\
\hline 0.18000 & 0.79487 & 0.500 & 0.900 \\
\hline 0.25000 & 0.64103 & 0.500 & 0.500 \\
\hline 0.32000 & 0.46154 & 0.500 & 0.100 \\
\hline 0.38000 & 0.28205 & 0.500 & 0.100 \\
\hline 0.20000 & 0.92308 & 0.500 & 0.900 \\
\hline 0.27000 & 0.76923 & 0.500 & 0.900 \\
\hline 0.33000 & 0.56410 & 0.500 & 0.500 \\
\hline 0.40000 & 0.41026 & 0.500 & 0.100 \\
\hline 0.47000 & 0.25641 & 0.500 & 0.100 \\
\hline 0.62000 & 0.82051 & 0.100 & 0.900 \\
\hline 0.70000 & 0.61538 & 0.100 & 0.900 \\
\hline 0.76000 & 0.46154 & 0.100 & 0.500 \\
\hline 0.83000 & 0.28205 & 0.100 & 0.100 \\
\hline 0.92000 & 0.10256 & 0.100 & 0.100 \\
\hline 0.70000 & 0.69231 & 0.100 & 0.900 \\
\hline 0.77000 & 0.48718 & 0.100 & 0.900 \\
\hline 0.85000 & 0.30769 & 0.100 & 0.500 \\
\hline 0.92000 & 0.15385 & 0.100 & 0.100 \\
\hline 1.00000 & 0,00000 & 0.100 & 0.100 \\
\hline
\end{tabular}

IFDNN is flexible and allows important neural network parameters such as network structure, learning rate, momentum, and error tolerance to be adjusted as required for a particular application. The results of neural network training may be saved in a format suitable for use by the real-time software RT_IFDNN. RT_IFDNN is written especially to perform the real-time incipient fault detection. It implements only the testing portions of the IFDNN software; no training is performed by RT_IFDNN. The fault detector is enhanced by its ability to collect on-line motor data from an analog to digital I/O board and to display results of the neural network calculations on the computer screen in real time.

\section{Results AND Discussion}

Thirty-five training and 70 testing data patterns have been collected according to a uniform distribution over the operating conditions under consideration on the laboratory test stand shown in Fig. 1. The laboratory test stand was configured so that possible combinations of stator winding and motor-bearing condition were represented in the training data set. The severity of the faults, and hence the relative condition of the stator windings and motor bearings, was evaluated using the thermal and efficiency criteria, respectively, as mentioned in Section II. For completeness, the numerical data relating $N_{c}$ and $B_{c}$ to $I$ and $\omega$ are shown in Table $I$.

Several conventional and higher-order feed-forward neural networks with three layers and different number of hidden 
neurons were trained using the laboratory training data set and the back-propagation algorithm implemented in the IFDNN software. All the data are normalized in the interval $[0,1]$. Define the two-norm error as

$$
\sqrt{\frac{1}{m}\left(\sum_{i=1}^{m} e_{i}^{2}\right)}
$$

where $e_{i}$ is the difference between the neural network output and the desired output of $i$ th training example and $m$ is the number of training examples ( $m=35$ in this project). Then both the conventional and higher order neural networks were trained until the average two-norm error of the entire training data set was less than or equal to 0.005 or until the absolute values of the change of total network weights was less than 0.0001 . Fig. 6 shows the training error as a function of training iterations for the conventional and higher order network structures - a training iteration being a pass-through the entire training data set to adjust the network weights. All the trained networks give $100 \%$ correct prediction for the training data, using the nearest neighborhood classification method mentioned in Section II.

The testing performance of the conventional and higher order networks are shown in Figs. 7 and 8, respectively. Conventional neural networks consisting of $4,8,12$, and 16 hidden nodes in their hidden layers were found to provide good performance in the prediction of stator winding condition $N_{c}$ of the induction motor. Accuracies as high as $98.7 \%$ were consistently achieved for all of the conventional neural networks tested. The conventional neural networks did not, however, provide satisfactory performance in the prediction of rotor bearing condition $B_{c}$. Accuracies of only $94.7 \%$ or less were recorded for the rotor-bearing condition-detection tests.

On the other hand, higher order neural networks consisting of $4,8,12$, and 16 hidden nodes in their hidden layers provided good performance in the prediction of both the stator-winding and bearing conditions. Accuracies of $98.7 \%$, the same level as conventional networks, were recorded for all tests of $N_{c}$. The higher order neural networks, however, also provided improved performance in the prediction of rotor-bearing condition. Accuracies of up to $97.3 \%$ were achieved, with the best performance achieved by the network containing 16 hidden nodes in its hidden layer. Also, all of the higher order neural networks, except the network containing 4 hidden nodes in its hidden layer, provided satisfactory results of better than $95 \%$ accuracy. It is believed that the network containing 4 hidden nodes in the hidden layer was not sufficient to accurately represent the characteristics of the data.

\section{Conclusions}

The objective of this paper has been to develop a neural network-based incipient fault detector that provides an accurate, real-time indication of the presence of incipient faults in ac induction motors. The higher order neural network incipient fault detectors consistently provided accuracy as good as or better than that of the conventional neural network

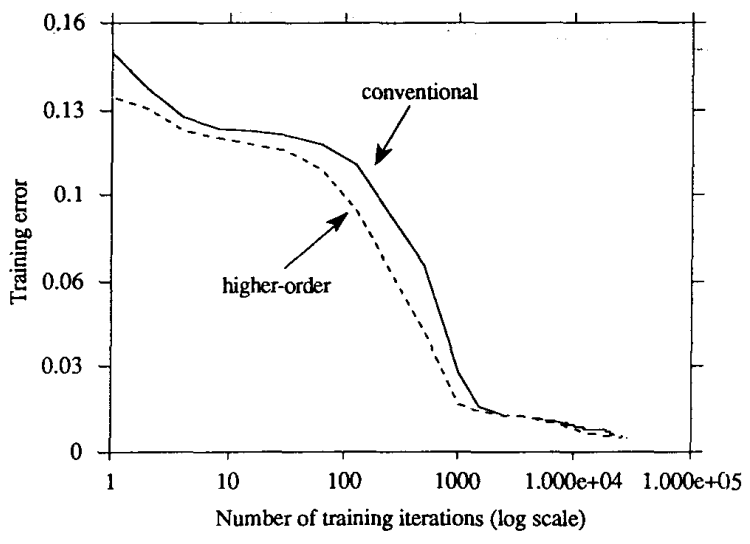

Fig. 6. Training error as a function of training iterations for the conventional (two inputs) and higher order (five inputs) IFDNN.

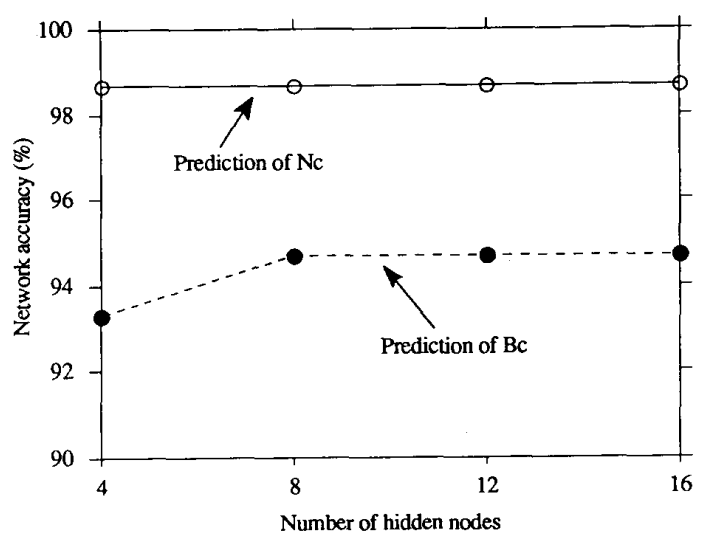

Fig. 7. Network performance of the conventional IFDNN (two inputs) as a function of hidden nodes.

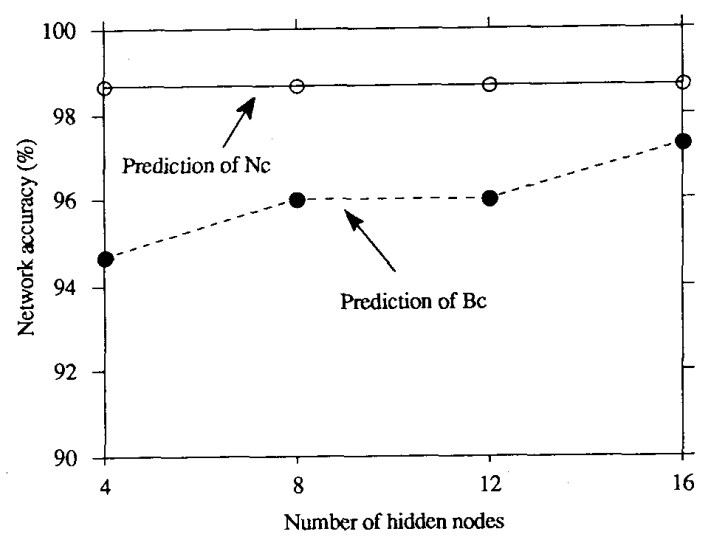

Fig. 8. Network performance of the higher order IFDNN (five inputs) as a function of hidden nodes. 
detectors. The results obtained using the higher order neural networks containing more than four units in the hidden layer provides a high level of accuracy, greater than $95 \%$, which is satisfactory for real-world applications.

\section{ACKNOWLEDGMENTS}

The authors would like to thank the reviewers of this paper for their valuable comments and suggestions.

\section{REFERENCES}

[1] P. J. Tavner and J. Penman, Condition Monitoring of Electrica Machines. New York: Research Studies Press Ltd., Wiley, 1989.

[2] S. Cambrias and S. A. Rittenhouse, Generic Guidelines for Life Extension of Plant Electrical Equipment, Electric Power Research Institute Report EL-5885, July 1988.

[3] M. Chow and S. O. Yee, "Application of neural networks to incipient fault detection in induction motors," J. Neural Network Comput., vol. 2, no. 3, pp. 26-32, 1991.

[4] M. Chow, "Artificial neural network methodology in real-time incipient fault detection of rotating machines," in Proc. National Science Foundation Workshop on Artificial Neural Network Methodology in Power Systems Engineering, Clemson University (Clemson, SC), April 8-10, 1990, pp. 80-85.

[5] D. R. Boothman, E. C. Elgar, R. H. Rehder, and R. J. Woodall, "Thermal tracking - A rational approach to motor protection," IEEE Trans. PAS, Sept./Oct. 1974, pp. 1335-1344.

[6] R. W. Smeaton, Motor Application and Maintenance Handbook, 2nd ed. New York: McGraw-Hill, 1987.

[7] D. E. Rummelhart and J. L. McClelland, Parallel Distributed Processing. Cambridge, MA: The MIT Press, 1986.

[8] R. P. Lippmann, "An introduction to computing with neural nets," IEEE ASSP Mag., Apr. 1987, pp. 4-22.

[9] P. J. Werbos, "Beyond regression: New tools for prediction and analysis in the behavioral sciences," Doctoral dissertation, Dept. of Applied Mathematics, Harvard University, Cambridge, MA, Aug. 1974. 